\title{
Fixed Eruption in Deeply Pigmented Subjects : Clinical Observations on 350 Patients
}

\author{
S. G. BROWNE, * M.D., F.R.C.P., F.R.C.S., D.T.M.
}

Brit. med. F., 1964, 2, 1041-1044

Ever since Brocq (1894) directed attention to certain druginduced eruptions that he called "fixed," in the sense that they were subject to recurrent exacerbation on each subsequent administration of the inducing drug, interest in the subject has increased. Among the many and varied syndromes provoked by new drugs and food additives (Sternberg and Bierman, 1963), fixed eruptions are becoming increasingly common (Welsh, 1961 ; Welsh and Ede, 1961).

The present study is based on 350 deeply pigmented patients suffering from fixed eruptions seen during the past 10 years (171 Congolese (Browne, 1959, 1960a, 1960b), 175 Nigerians, and 4 others), and provides data that may shed some light on the continuing problem of the localization of the eruptive elements.

\section{Primary Sensitization}

In the great majority of cases it was possible with a reasonable degree of probability to determine the drug responsible for the induction of the fixed eruption. The following drugs were incriminated:

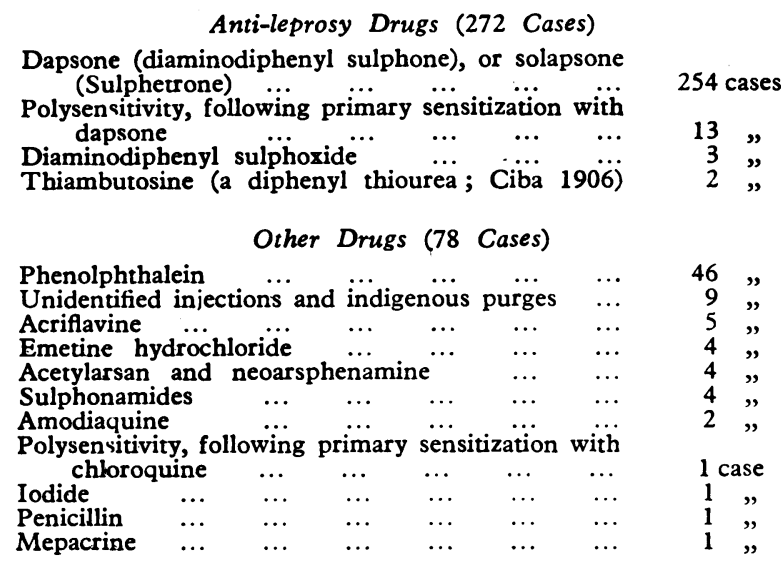

The fact that in 272 patients (over three-quarters of the total) anti-leprosy drugs were responsible for primary sensitization reflects the number of leprosy patients on prolonged treatment under my care, rather' than any special tendency of such drugs to induce fixed eruptions. Similarly, the preponderance of phenolphthalein as a primary sensitizer in the general population in Nigeria indicates the wide availability of numerous proprietary purgative preparations containing this substance. The incidence of fixed drug eruptions in the population at risk in Nigeria is in the nature of things quite unknown in respect of self-administered medication, and also in respect of drugs nominally given under medical supervision.

Accurate figures are available regarding 5,349 patients under treatment for leprosy in the Yakusu medical area in the ex-Belgian Congo ; 160 (approximately $3 \%$ ) of these patients had fixed eruptions caused either by dapsone given orally or by intramuscular injection as a $25 \%$ oily suspension, or by

* Leprosy Service Research Unit, Uzuakoli, Eastern Nigeria. solapsone given intramuscularly as a $50 \%$ aqueous solution. In Nigeria the three cases in which diaminodiphenyl sulphoxide was responsible for primary sensitization occurred in a total of 122 patients receiving the drug (Browne and Davey, 1961). In view of the widespread use of sulphonamides and penicillin, the small number of fixed eruptions induced by these drugs probably indicates their low capacity for primary sensitization in deeply pigmented subjects. The absence of sedatives, tranquillizers, and soporifics in this list of primary sensitizers is a reminder that these drugs have not yet come into widespread use in the two countries.

Of the 175 patients with fixed eruptions seen in Nigeria, 56 attended the diagnostic clinic of the Leprosy Service Research Unit, Uzuakoli, suspecting that the rash was a sign of leprosy. They represented about $8 \%$ of patients coming to the clinic who did not have leprosy, or about $4 \%$ of the total attending. In 300 patients at Ibadan, Nigeria, Harman (1962) found 3\% with fixed eruptions. Gelfand (1959) records three cases of "melanosis" from Mashonaland, emphasizing the rarity of the condition, but hazards no suggestion concerning a possible cause. Marshall (1963), in South Africa, is familiar with fixed eruptions induced principally by phenolphthalein and the sulphonamides. Chargin and Leifer (1940) found that negroes were more susceptible to drug-induced fixed eruptions than were Caucasians. Of the 56 patients in Nigeria, in no fewer than 36 the eruption was induced by phenolphthalein. Other drugs were: dapsone (five), acetylarsan (three), sulphathiazole (two), indigenous purges (two), iodide (one), neoarsphenamine (one), and unidentified agents (six). As it happened, none of the five patients who had been treating themselves with dapsone for suspected leprosy actually had leprosy; they all presented hypopigmented mycotic lesions of the skin together with the typical hypermelanotic macules of the fixed eruption. The "cure" was more cosmetically obvious than the disease, and less amenable to treatment.

\section{Nature and Colour of the Lesions}

Whatever the precipitating cause the characteristic lesions in this series were essentially similar. They consisted of $(a)$ welldefined blackish macules, mostly round or oval and varying in size from 0.5 to $10 \mathrm{~cm}$. or even $15 \mathrm{~cm}$. in diameter, the majority being 2 to $4 \mathrm{~cm}$. in diameter; and $(b)$ a diffuse hypermelanosis occupying extensive areas of the face, trunk, and limbs, and in rare cases involving almost the entire body surface. Most patients had either one or other variety of lesion; a few had both.

The colour of the lesions in the intact dark-brown skin was black, and the intensity of the blackness tended to become deeper with each succeeding exacerbation. In the lighter-hued skin the colour varied from purplish-grey to slate-grey. Where the overlying epithelium had been lost by reason of vesiculation, bullae formation, or rubbing or scratching of the itching skin, the colour varied from slate-grey to sky-blue, depending on the amount of melanin remaining in the basal layer and the amount and dispersion of particulate melanin in the dermis (the scattering effect, or Tyndall phenomenon). 


\section{Symptomatology}

The classical course of localized or generalized dermatitis, often papular $\mathrm{r}$ morbilliform, followed a week or so later by discrete hyperpigmented macules or generalized hypermelanosis, with precocious recurrence of the irritation after each administration of the offending drug, was observed in most cases. Successive exacerbations tended to become progressively more serious. Vesicles and weals appeared in the macules, followed by desquamation; new lesions appeared at hitherto unaffected sites.

Systemic symptoms varied greatiy in intensity, from a fleeting malaise occurring simultaneously with each exacerbation of the fixed eruption, to severe prostration, with raised temperature ("drug fever"), nausea, diarrhoea, and cramp-like abdominal pains. Apart from local irritation in the skin lesions, most patients had no symptoms. In these rather phlegmatic peasantfarmers the psychological component was apparently small, and no instance of exacerbation could be attributed to it. After Jadossohn's (1905) amplification of the conception of the fixed cruption to include recurrent extracutaneous manifestations of sensitivity, the occurrence may be mentioned of slight headache and insomnia accompanied by generalized pruritus after each dose of dapsone, or bouts of transient euphoria sometimes progressing to mild mania. A few patients complained of giddiness occurring three hours after each dose of dapsone, and one of them noted that the giddiness occurred only in bright sunlight. Two patients in whom insomnia recurred after each dose of dapsone developed a psychosis necessitating prolonged interruption of anti-leprosy treatment. (These patients with extracutaneous periodic disturbances associated with ingestion of drugs are not included in the totals of fixed eruptions given in this paper).

\section{Atypical Features Observed in this Series}

In a relatively high proportion of cases there were departures from this characteristic sequence. An itching papular rash appeared in some cases almost immediately after the first dose of the drug, and the fixed eruption followed within a few days. In 10 patients the fixed eruption appeared after a single dose of dapsone as low as $25 \mathrm{mg}$. and not exceeding $100 \mathrm{mg}$. and in nine patients it appeared after the first dose of solapsone ( 250 $\mathrm{mg}$.). In most cases it was possible to ascertain that the drug had never been taken previously, and the occurrence of parallergic sensitization by a chemically related drug-that is, a sulphonamide sensitizing the subject to a sulphone-could be virtually excluded by giving the patient a provocative dose of each drug separately. At the other extreme, cutaneous sensitivity suddenly appeared in three patients after seven years of dapsone therapy and in two after six years. About half the patients experienced the first eruption in the second year of treatment. If drugs like phenolphthalein were taken by the general population with the same frequency and regularity as sulphones are administered to leprosy patients, it is an open question what proportion of them would become sensitized to the drug.

Once the fixed eruption appeared, irritation in the lesions might follow oral ingestion of the drug (or the placing of a small test dose under the tongue) after as short an interval as a minute, or it might be delayed for several hours. Some subjects made no complaint of irritation in the typical lesions before their appearance, as they developed, or on taking further doses of the drugs. While subjective appreciation of cutaneous sensation may vary between the phlegmatic and the choleric, the slow-witted and the sensitive, the range in both subjective complaints and visible localized papular dermatitis was very marked in this series. The itching might be felt indiscriminately in the hyperpigmented macules and in the apparently normal skin, or vice versa ; or, alternatively, both in skin showing papular and pigmentary changes and in normal skin. Rarely, the nasal mucosa might irritate and discharge quantities of pituita simultaneously with each exacerbation of the fixed eruption. Discrete or generalized irritation might recur regularly, in the absence of any papular or hyperpigmented eruption.

Departures from the usual sequence of progressive exacerbation on repeated administration of the causative drug were numerous. Notwithstanding the presence of a fixed eruption in leprosy patients, treatment for the underlying disease was of course necessary, either with the same drug or with another sulphone, or with a drug of dissimilar chemical constitution. When, for instance, treatment with twice-weekly oral doses of dapsone was continued, no change might occur in the lesions of the fixed eruption, or there might even be a gradual lessening of the intensity of the hypermelanosis until it eventually disappeared after perhaps 18 to 24 months. This prolonged refractory or negative phase was much commoner than a short negative phase followed by a return of the sensitivity to its previous level or to an enhanced level, as shown by appearance of many new lesions when the drug was readministered.

Serious exacerbations noted in this series include one case of exfoliative dermatitis and two of toxic epidermal necrolysis, one due to dapsone and the other to phenolphthalein (Browne and Ridge, 1961). There was no case resembling the StevensJohnson syndrome complicating erythema multiforme.

\section{Localization of the Skin Lesions}

In 145 Congolese patients whose fixed eruptions were occasioned by dapsone or solapsone the countable discrete hypermelanotic macules were distributed as shown in the Table.

Most workers remark on the apparently haphazard distribution of the lesions of fixed eruptions in the skin, and on the impossibility of discovering any one factor that might explain this localization. In some series the mouth, the genitalia, and the sacral region appear to be sites of predilection, and the verge noire of Fournier has become classic. In the present series, discrete lesions often appeared in the same bilaterally symmetrical regions of the skin (particularly the deltoid, the scapular, the abdominal, and the adductor regions, and the inner aspect of the arms), and individual lesions within these areas might correspond exactly with lesions on the opposite side of the body. On the thorax multiple lesions sometimes followed the line of the intercostal nerves. Some trunk lesions were centrally situated, straddling the midline symmetrically. In the case particularly of the ears and lips and superciliary region, and less noticeably elsewhere on the face, well-defined

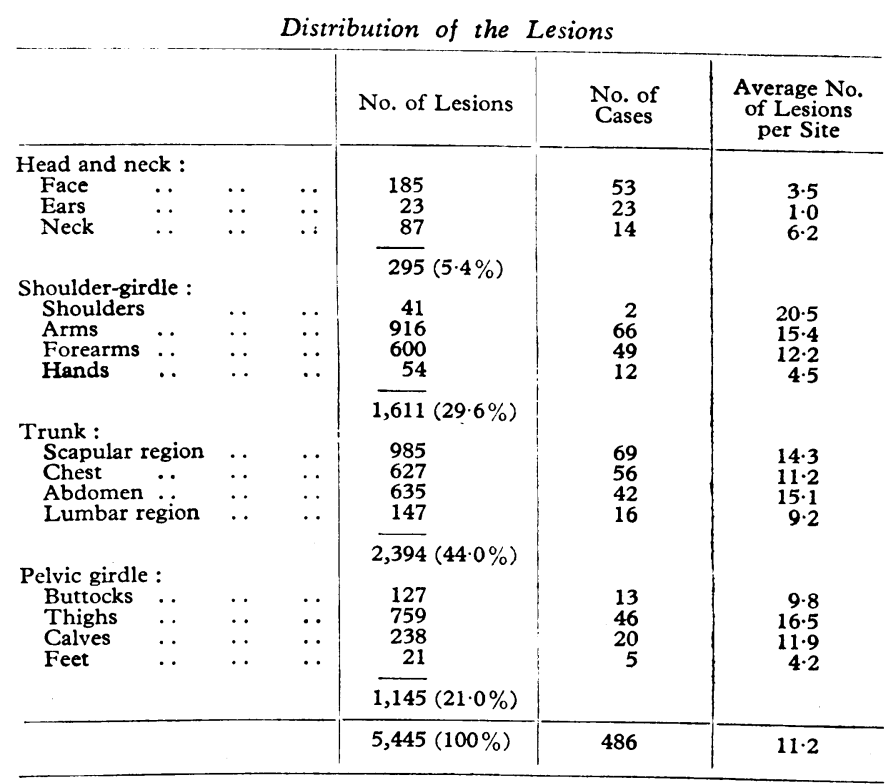


hypermelanotic macules were often found to be strictly symmetrical, to within a millimetre.

Mucosal Lesions.-In the 145 Congolese patients referred to above, together with 15 patients in whom the fixed eruption was diffusely distributed over the face and trunk, hypermelanotic lesions appeared in the mucosae of 82 cases in the following situations: lips in 62, cheeks in 16, hard palate in 2 , gums in 1 , tongue in 1 . In most instances the mucosae were involved continuously with the adjacent skin. The range of pigmentation of the buccal mucosa in deeply pigmented subjects varies enormously, and it may be that the incidence of mucosal lesions was in reality greater than indicated above. In two Nigerian children hyperpigmented fixed eruptions in the periorbital region encroached upon the palpebral conjunctivae. In several cases macules on the upper lip and nose were continuous with prolongations into the mucosa of the vestibule. No lesions were seen on the urethral mucosa.

Fixed Eruptions in Nails.-Apart from the single case of mepacrine, and the five cases due to intravenous medication with $3 \mathrm{ml}$. of $1 \%$ aqueous acriflavine, there was no example of nail lesions in this series. Mention may be made in this connexion of the four cases of fixed eruption due to repeated intramuscular injections of $40 \mathrm{mg}$. of emetine hydrochloride. Recurrent papular dermatitis confined to the skin of the fingertip pulp and the dorsal surface of the ungual phalanx was not followed by pigmentary changes in the nail but by hypermelanosis and desquamation of the affected skin.

Hair.-No case of change of hair colour was observed in association with fixed eruption of the skin.

Diffuse Hypermelanosis. - In addition to the symmetrically disposed discrete lesions mentioned above, in about one-tenth of the subjects the lesions were diffuse and symmetrical. These occurred particularly on the face, but some subjects presented a diffuse hypermelanosis of the face and trunk and perhaps of the limbs as well. When the skin of the trunk was generally hypermelanotic, the only areas spared were the inguinal region, a horizontal band in the lumbo-sacral region, and the axillae. Diffuse hypermelanosis of the face often conformed to certain distinct patterns, recalling chloasma uterinum (Sellei, 1930) and suggesting possible mediation of the melanocyte-stimulating hormone of the pituitary (Brit. med. F., 1961): (a) The periphery of the face-that is, forehead, ears, preauricular regions, chin, and avoiding the nose, cheeks, and lips. (b) The most prominent parts of the face-that is, the forehead, the cheeks, the nose, the chin. (c) The circumoral and periorbital zones. (d) The whole of the face except the circumoral and periorbital zones. (e) The beard area.

Fixed Eruptions in Children.-Some workers have found a much lower prevalence of fixed eruptions in children. This may be due wholly or in part to opportunities of exposure to the inducing drugs. In the Congo series of 160 patients with sulphone-induced fixed eruptions eight were in children, representing $0.6 \%$ of those under treatment for leprosy and $0.04 \%$ of the population. The corresponding figures for men were $94,4.3 \%$, and $0.64 \%$; for women, $58,3.0 \%$, and $0.47 \%$. Of the 36 examples of phenolphthalein-induced fixed eruption seen in Nigeria three were in children under 16.

\section{Observations on some of the Sensitizing Drugs}

Dapsone.-Lowe (1962) noted that breast-fed infants whose mothers were taking dapsone for leprosy sometimes developed a diffuse hypermelanosis of the periorbital regions, the lips, the palms, and elsewhere. The hyperpigmentation subsided, to disappear completely if dapsone treatment of the mother was suppressed or if the child was weaned. We have noted a similar occurrence with dapsone and also with B 663 (Geigy) (Browne and Hogerzeil, 1962). One case of fifth-week dapsone dermatitis (due to self-medication) occurred in this series. Such cases were often seen during early high-dosage dapsone therapy ; they were characterized by high temperature, prostration, toxaemia, exfoliative dermatitis, hepatitis, and severe anaemia, and were often fatal (Allday and Barnes, 1951).

Phenolphthalein.-Lewin (1962) and others have suggested that systemic symptoms attributable to phenolphthalein may be rare because not only is the drug poorly absorbed from the intestine but its carthartic action may render absorption even poorer. In this series abnormally violent purgation often followed medicinal doses of phenolphthalein; damage to the intestinal mucosa may have facilitated absorption of the drug.

Sulphonamides incriminated were: sulphathiazole (three cases) and sulphaguanidine (one case) (Browne, 1960b).

Iodide.-The only case in this series was due to potassium iodide in a proprietary "blood mixture." Fixed eruptions induced by iodide are apparently very rare (Baker, 1962).

Unidentified Agents.-Nine patients presented themselves with the characteristic history of recurrent irritation of hypermelanotic macules, and admitted having received unknown illicit injections or purgative draughts made from various roots, barks, and leaves. It was not possible to identify these agents.

\section{Polysensitivity}

In addition to the specific reaction consequent on repeated administration of the same drug, several instances of group reaction were observed in which closely related chemical compounds-for example, other sulphones-or more distantly related compounds-for example, sulphonamides-were responsible for exacerbation in dapsone-induced fixed eruptions. There were also some instances of polyvalent or heterophilic reaction, sometimes termed "polyallergic sensitization," in which drugs of totally different chemical structure precipitated exacerbations precisely similar to those caused by the inducing drug.

Thirteen adult patients (eight men and five women) who had developed fixed eruptions while receiving standard dapsone treatment for leprosy and who were given other anti-leprosy drugs experienced recurrence of all the symptoms and signs of the fixed eruption with every dose of the new drugs. Thus four of these patients also proved sensitive to thiambutosine; two to thiacetazone; two to diaminodiphenyl sulphoxide; one was sensitive to solapsone, thiambutosine, sulphadimidine, and to antihistaminics and barbiturate analgesics; two others were sensitive to all the above anti-leprosy drugs, and to ditophal (Etisul), barbiturates, and antihistaminics.

No case of primary sensitization to ditophal was encountered in about 200 patients treated with this drug. Contact dermatitis on inunction and generalized pruritus after localized percutaneous absorption are reported to be not uncommon in light-skinned patients.

The only instance of polysensitivity encountered in a patient not suffering from leprosy in this series was a boy of 6 who, having developed a typical fixed eruption while taking chloroquine, experienced the same sequence of localized irritation and exacerbation of the fixed eruption after every dose of amodiaquine (Tuffanelli et al., 1963).

\section{Desensitization}

Desensitization for dapsone dermatitis was attempted in 52 patients who suffered from a generalized itching papular eruption on each administration of sulphone for leprosy (Browne, 1963). In 48 it was successful.

A similar desensitizing procedure may be successfully applied in the case of patients suffering from lepromatous leprosy in whom recurrent reactional episodes appear to be precipitated by standard doses of anti-leprosy drugs. Once the patient is in this delicate hypersensitive state, diverse endogenous as well as extraneous factors may cause fresh outbreaks of erythema nodosum leprosum, together with exacerbation of the cutaneous 
lesions of lepromatous leprosy and recrudescence of such presumably allergic manifestations as iritis, iridocyclitis, and polyneuritis. Whatever the precise mechanism of the action of anti-leprosy drugs in precipitating such recurrences, slow desensitization by standard methods as for dapsone dermatitis may be the only procedure permitting resumption of leprosy treatment.

\section{Patch-testing}

Several tests were carried out with contact patches, and also scratch tests employing aqueous and alcoholic solutions of the suspected drug, but in view of the equivocal results obtained they were abandoned. All subjects did not react to patch tests of the drug to which they were shown to be sensitive by oral administration, and in some patients polysensitivity and nonspecific sensitization occurred. Where the histury was uncertain, or where the patient had taken more than one drug, it was generally possible, by oral provocation, to identify the drug responsible for the fixed eruption despite the theoretical possibility of polysensitization on the one hand and a refractory phase on the other.

\section{Non-specific Exacerbating Factors}

Although these have been reported by other workers, nonspecific factors in food and drink, and physical factors such as cold and heat, were not suspected of precipitating exacerbation of the fixed eruptions in the present series. However, some cases of recurrent spontaneous exacerbation of the lesions might have been attributable to endogenous factors derived from tissue breakdown, as in the type of erythema multiforme that recurs with a periodicity of several days. Photosensitivity appeared to play no part in recurrent exacerbations.

\section{Discussion}

The following points of interest emerge from the study of this series.

1. The variability of the symptomatology and natural history of the fixed drug eruption in deeply pigmented subjects. The fixed eruption, with its typical discrete lesions undergoing recurrent exacerbation, merges imperceptibly into a diffuse type of postinflammatory hypermelanosis that may follow a widespread druginduced itching papular eruption. In both kinds of lesion discrete or generalized irritation may precede or accompany the appearance of the pigmentary changes. When a drug is given orally twice a week, the low-grade irritation may be of no moment and the hypermelanosis seems to deepen gradually, unpunctuated by periods of exacerbation. To generalize, the nearer the eruption approaches the classical description of discrete lesions in the skin the more typical is the sequence of recurrent exacerbation, and the more diffuse the hypermelanosis the less marked is the periodicity of signs of exacerbation.

2. The high proportion-namely, up to $3 \%$ - of deeply pigmented subjects who develop fixed eruptions on prolonged treatment with sulphones. In general, deeply pigmented suhjects seem especially prone to react to cutaneous irritation by hypermelanosis. In southeast India the corresponding incidence is less than $0.1 \%$.

3. The not infrequent appearance of fixed eruptions after a single dose of a drug.

4. The gradual decrease in intensity of the hypermelanosis of the fixed eruption in some patients and even its eventual disappearance despite the continued administration of the incriminated drug.

5. The two groups into which the eruptions tend to fall: asymmetrical and symmetrical, often corresponding to discrete and diffuse.

Certain parallels between fixed eruptions and leprosy suggest themselves. Firstly, it may be difficult or impossible to differentiate on sight the diffuse type of fixed eruption from the hyperpigmentation of the granuloma-infiltrated lepromatous skin occurring after medication with dapsone or amodiaquine (Doull, 1959 ; Browne, 1961), or the aposafranin B 663 Geigy (Browne and Hogerzeil, 1962). Secondly, the apparent haphazard localization of tuberculoid lesions in leprosy, which is associated with local tissue hypersensitivity, may resemble the asymmetrical type of fixed eruption. Weddell et al. (1963) have shown that the local cutaneous nerves may be symmetrically involved in leprosy while obvious skin lesions may be present on one side only. In lepromatous leprosy (macular or nodular), widespread lesions of the skin, symmetrically disposed, show certain similarities to the diffuse type of fixed eruption. Thirdly, the areas spared in confluent macular lepromatous leprosy - that is, the inguinal region, the lumbo-sacral region, and the axillae-are precisely those that may be spared in the generalized hypermelanosis of the diffuse type of fixed eruption.

These observations may throw some light on the apparent discrepancies in passive transfer experiments of sensitized skin reported by Naegeli et al. (1930) and by Wise and Sulzberger (1933). The autotransplants may retain their hypersensitivity indefinitely or may lose it within a short time. The reason for these differences seems to reside in the precise level of dermal tissue that bears the brunt of the sensitization. The same factor also determines the level of eventual cleavage in epidermis or dermis resulting in exfoliative dermatitis or toxic epidermal necrolysis.

\section{Summary}

Fixed eruptions induced principally by dapsone and phenolphthalein are not uncommon in deeply pigmented African subjects, up to $3 \%$ of leprosy patients developing sulphone-induced eruptions.

While the majority of fixed eruptions conform to the classical pattern, many show departures therefrom in symptomatology and natural history of the lesions. In particular, typical lesions may show no exacerbation with continued administration of the inducing drug, and may even regress and disappear. The commoner asymmetrical discrete lesions are contrasted with strictly symmetrical facial lesions and with a symmetrical diffuse hypermelanosis. Some examples of the latter merge imperceptibly into diffuse post-inflammatory hypermelanosis.

Certain parallels are suggested between these two polar types of fixed eruption and the polar types of leprosy-tuberculoid and lepromatous.

My thanks are due to Dr. S. O. Egwuatu, Chief Medical Officer, Ministry of Health, Eastern Nigeria, for permission to publish this article.

\section{REFERENCES}

Allday, E. J., and Barnes, J. (1951). Lancet, 2, 205.

Baker, H. (1962). Brit. 7. Derm., 74, 310.

Brtt. med. 7., 1961, 2, 634.

Brocq, L. (i894). Ann. Derm. Syph. (Paris), 5, 308.

Browne, S. G. (1959). Trans. roy. Soc. trop. Med. Hyg., 53, 495. - (1960a). Leprosy Rev., 31, 54.

(1960b). Brit. med. 7., 1, 621 .

(1961). Int. F. Leprosy, 29, 107

(1963). Brit. med. 7., 2, 664.

and Davey, T. F. (1961). Leprosy Reu., 32, 194.

and Hogerzeil, L. M. (1962). Ibid., 33, 6.

and Ridge, E. (1961). Brit. med. $\dddot{\xi} ., 1$, 550.

Chargin, L., and Leifer, W. (1940). F. invest. Derm., 3, 443.

Doull, J. A. (1959). Int. F. Leprosy, 27, 385.

Gelfand. M. (1959). Cent. Afr. F. Med., 5, 595.

Harman, R. R. M. (1962). Brit. F. Derm., 74, 416.

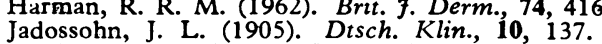

Lewin, K. J. B. (1962). Lancet, 2, 461 .

Lowe, J. (1952). Leprosy Rev., 23, 4.

Marshall, J. (1963). Arch. Derm., 87, 419.

Naegeli, O., de Quervain, F., and Stalder, W. (1930). Klin. Wschr., 9,

Sellei, J. (1930). Derm. Z., 58, 17.

Sternberg, T. H., and Bierman, S. M. (1963). Arch. Derm., 88, 779.

Tuffanelli, D., Abraham, R. K., and Dubois, E. L. (1963). Ibid., 88, 419.

Weddell, G., Palmer, E., Rees, R. J. W., and Jamison, D. G. (1963).

The Pathogenesis of Leprosy, edited by $\mathrm{V}$. R. Khanolkar. Ciba Foundation Study Group No. 15. Churchill, London.

Welsh, A. L. (1961). The Fixed Eruption. Blackwell, Oxford.

Wis and Ede, M. (1961). Arch. Derm., 84, 1004.

Wise, F., and Sulzberger, M. B. (1933). Ibid., 27, 549. 